\title{
Qualifikation, Kompetenzentwicklung und Professionalisierung im Dienstleistungssektor
}

Über ein Jahrhundert hat in Deutschland die industrielle Produktion die gesellschaftliche Wertschöpfung dominiert. Deutschland ist bis in die Gegenwart hinein in seinen Arbeitsinstitutionen Industriegesellschaft par excellence. Das kompakte industrielle Produktionsmodell hat das Verständnis von Arbeit in der Gesellschaft ebenso tief greifend geprägt wie die Formen der betrieblichen Arbeitsorganisation, der Berufsausbildung und der industriellen Beziehungen. Auch wenn Deutschland in Zukunft eine stark industriebasierte Ökonomie bleiben wird, wird die Erwerbstätigenstruktur immer mehr von den Dienstleistungsbereichen bestimmt sein und der Anteil der Industriebeschäftigten auf weniger als ein Viertel zurückgehen. Der Artikel vertritt die These, dass für Dienstleistungsökonomien ein neuer Typ von Erwerbsarbeit, der andere Merkmale als industrielle Herstellungsarbeit aufweist, strukturbestimmend wird: interaktive Arbeit. Damit stellen sich neue Fragen zur Arbeitsorganisation und Professionalisierung dieses neuen Typs von Arbeit, die im Artikel mit Blick darauf abgehandelt werden, welche Herausforderungen sie für Dienstleistungsgewerkschaften beinhalten.

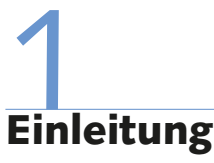

Wie ein roter Faden durchzieht seit Jahrzehnten die wissenschaftliche Diskussion der Satz von der Unmöglichkeit, eine umfassende positive Definition für Dienstleistungsarbeit zu formulieren, die das allen Dienstleistungstätigkeiten Gemeinsame in einem ähnlich kompakten ArbeitsregimeKonzept fasst, wie es ein Jahrhundert lang für die industrielle Produktionsarbeit mit ihrer klar definierbaren Produktivität, ihrem Technik- und Rationalisierungsbezug und ihrer Facharbeitskonzeption möglich war. Die Bedingung für das Industriearbeits-Paradigma, welches lange Zeit die Vorstellungen von Erwerbsarbeit in Deutschland dominierte, war jene relative Einheitlichkeit in den Arbeitsformen und Entwicklungspfaden (Qualitätsproduktion), die branchenspezifische Differenzen überformte und die nicht zuletzt auch die Bündelung von Arbeitsinteressen in den Industriegewerkschaften ermöglichte. Eine solche relative Einheitlichkeit ist für Dienstleistungsarbeit aufgrund ihrer inhaltlich-funktionalen Heterogenität wie auch ihrer institutionellen Differenziertheit (teils in privatwirtschaftlicher, teils in staatlicher, teils in Non-Profit-Organisation oder in Selbstständigkeit) weder gegeben noch herstellbar.

Der Artikel geht in drei Argumentationsschritten der Frage nach, inwieweit es in dem riesigen Feld der Dienstleistungsberufe (ca. 80 \% der Gesamtbeschäftigten) jenseits der Heterogenität (Abschnitt 2) Gemein- samkeiten in den Arbeits- und Kompetenzstrukturen gibt (Abschnitt 3) und was diese für die Professionalisierung der Dienstleistungsarbeit bedeuten können (Abschnitt 4).

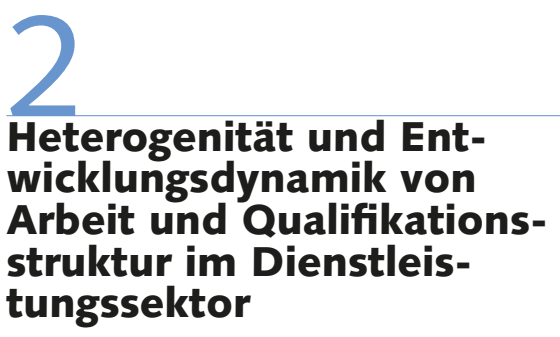

Die doppelte - funktionale wie institutionelle - Heterogenität der Dienstleistungstätigkeiten macht übergreifende Aussagen zu Qualifikation, Kompetenz und Professionalisierung sehr schwierig. Um einen Eindruck von der inhaltlichfunktionalen Heterogenität zu gewinnen, werden im Folgenden die aktuell etwa 80 \% aller Erwerbstätigen, die in Dienstleistungsberufen arbeiten, in ihrer funktionalen Zuordnung zu Berufs(haupt)feldern und anschließend in ihrer Qualifikationsstruktur differenziert.

Abbildung 1 zeigt die Erwerbstätigenstruktur in Deutschland und ihre wahrscheinliche Entwicklung bis 2025 nach Berufshauptfeldern, die noch einmal in drei Sektoren - produktionsbezogene Berufe, primäre und sekundäre Dienstleistungsberufe $^{1}$ - gebündelt sind. Sie basiert auf einer komplexen Projektion des Arbeitskräftebedarfs bis 2025 einer gemeinsamen Forschergruppe des Bundesinstituts für Berufsbildung (BIBB) und des Instituts für Arbeitsmarkt- und Berufsforschung (IAB) (vgl. Helmrich/Zika 2010).
Die Grafik zeigt, dass die produktionsbezogenen Berufe nur noch gut ein Fünftel der Erwerbstätigen ausmachen und sich ihr Anteil weiter verringern wird auf knapp $18 \%$ 2025. Der zweite große Beschäftigungssektor, die primären Dienstleistungen, umfasst relativ einfache kaufmännische Tätigkeiten im Verkauf, Büro und Verwaltung sowie allgemeine

\footnotetext{
Die Differenzierung in primäre und sekundäre Dienstleistungen greift nach Klauder (1990, zitiert nach Hall 2007, S. 159f.) den Unterschied von Dienstleistungstätigkeiten auf, die (bei den primären Dienstleistungen) „im Schwerpunkt eine Verlängerung des Produktionsweges nach vorne und hinten dar(stellen), [...] den gesamtwirtschaftlichen "Produktionsfluss" aufrecht(halten) und direkt in den Konsum eingehen ". Demgegenüber umfassen die sekundären Dienstleistungen solche, die in der Regel immaterielle Güter herstellen, zumeist geistige Arbeit darstellen und, soweit sie auf die industrielle Produktion bezogen sind, diese qualitativ über Nutzung von Wissen und Verbesserung des Humankapitals fördern. Die Zuordnung zum Produktionsprozess der beiden Dienstleistungskategorien bei Klauder passt nicht ganz, da beide sowohl unmittelbar auf privaten Konsum als auch auf staatliche Infrastrukturleistungen bezogen sind. Es existieren andere funktionale Klassifikationen, z.B. "konsumbezogene" , "distributive", "produktionsnahe ${ }^{\prime}{ }_{\text {, }}$ soziale ${ }^{\prime}$, "staatliche ${ }^{\mu}$ Dienste u.a. (vgl. Hall 2007, S. 161), die aber statistisch bisher nicht so gut aufbereitet sind, sodass hier auf die Klassifikation „primäre“ vs. "sekundäre" Dienstleistungen zurückgegriffen wird.
}

Martin Baethge, Prof. Dr., ist Präsident des
Soziologischen Forschungsinstituts (SOFI) an
der Universität Göttingen. Arbeitsschwer-
punkte: Dienstleistungs- und Industrieso-
ziologie, Bildungs- und Berufssoziologie,
internationale Komparatistik.
e-mail:
martin.baethge@sofi.uni-goettingen.de


Abb. 1: Erwerbstätige 2005 bis 2025 nach Berufshauptfeldern - in \%, absolute Werte* in Tsd. in Klammern -

\begin{tabular}{|c|c|c|c|c|c|c|}
\hline Rohstoffgewinnende Berufe & $2,4 \quad(920)$ & \multirow{3}{*}{21,2} & 2,1 & \multirow{3}{*}{19,3} & 1,9 & \multirow{3}{*}{$\begin{array}{l}\text { Produktionsbezogene } \\
\text { Berufe } \\
17,9\end{array}$} \\
\hline Be-, verarbeitende u. instandsetzende Berufe & $\begin{array}{c}13,8 \\
(5.257)\end{array}$ & & 12,7 & & 11,7 & \\
\hline $\begin{array}{r}\text { Maschinen u. Anlagen steuernde } \\
\text { u. wartende Berufe }\end{array}$ & $5,0(1.915)$ & & 4,5 & & 4,3 & \\
\hline Berufe im Warenhandel, Vertrieb & $\begin{array}{c}11,0 \\
(4.189)\end{array}$ & \multirow{4}{*}{47,9} & 10,7 & \multirow{4}{*}{47,6} & 10,4 & \multirow{4}{*}{$\begin{array}{l}\text { Primäre } \\
\text { Dienstleistungsberufe } \\
47,6\end{array}$} \\
\hline $\begin{array}{l}\text { Verkehrs-, Lager-, Transport-, } \\
\text { Sicherheits-, Wachberufe }\end{array}$ & $\begin{array}{c}9,2 \\
(3.507)\end{array}$ & & 9,0 & & 8,8 & \\
\hline Gastronomie- u. Reinigungsberufe & $\begin{array}{c}10,4 \\
(3.954)\end{array}$ & & 11,5 & & 12,6 & \\
\hline Büro, kfm. Dienstleistungsberufe & $\begin{array}{c}17,4 \\
(6.630)\end{array}$ & & 16,4 & & 15,8 & \\
\hline Technisch- naturwissenschaftliche Berufe & 8,4 & \multirow{5}{*}{30,9} & 8,4 & \multirow{5}{*}{33,1} & 8,5 & \multirow{5}{*}{$\begin{array}{l}\text { Sekundäre } \\
\text { Dienstleistungsberufe } \\
34,5\end{array}$} \\
\hline $\begin{array}{l}\text { Rechts-, Management- u. wirtschaftswiss. Berufe } \\
\text { Künstl., Medien-, geistes u. sozialw. Berufe }\end{array}$ & $\begin{array}{ll}4,6 & (1.738) \\
3,0 & (1.138)\end{array}$ & & $\begin{array}{l}5,0 \\
3,4\end{array}$ & & $\begin{array}{l}5,3 \\
3,7\end{array}$ & \\
\hline Gesundheits- und Sozialberufe, Körperpflege & $\begin{array}{c}11,2 \\
(4.275)\end{array}$ & & 12,6 & & 13,5 & \\
\hline Lehrberufe & $3,6 \quad(1.418)$ & & 3,7 & & 3,6 & \\
\hline & 2005 & & 2015 & & 2025 & \\
\hline
\end{tabular}

*Bei den Absolutwerten handelt es sich um „Durchschnittswerte“, bei denen das jährliche Arbeitsstundenvolumen aller Erwerbstätigen des jeweiligen Berufshauptfeldes (Vollzeit-, Teilzeit-, geringfügig Beschäftigte) durch die Zahl der tatsächlichen Erwerbstätigen dividiert worden ist. Würde man die empirische Arbeitszeitstruktur zugrunde legen, würde sich die Zahl der realen Erwerbstätigen in den Berufsfeldern erhöhen.

Dienstleistungen, wie gastronomische Tätigkeiten, Reinigen, Transportieren, Lager- und Sicherheitstätigkeiten. Das Gewicht dieser Dienstleistungen wird für die nächsten 15 Jahre als relativ stabil angenommen, allerdings mit nicht unwichtigen Verschiebungen in den Anteilen zwischen den Berufshauptfeldern: die kaufmännischen Tätigkeiten nehmen ab (ca. -2,2 Prozentpunkte), während die Gastronomie- und Reinigungsberufe um die gleiche Größenordnung zunehmen (Abbildung 1). Man kann diese Verschiebung als Verstärkung der Tendenz zu personenbezogenen Dienstleistungen interpretieren, wobei die Anteilsverlagerung nicht allein aus der erhöhten Nachfrage nach personenbezogenen Dienstleistungen, sondern auch aus der weitergehenden Rationalisierung vor allem bei den Büro- und Verwaltungstätigkeiten aufgrund nicht zuletzt von Informations- und Kommunikations (IuK)Technologie zu erklären ist. Insgesamt wird an den vier Berufshauptfeldern die inhaltlich-funktionale Differenz der unter der Kategorie primäre Dienstleistungen zusammengefassten Tätigkeiten deutlich:
Was verbindet die Tätigkeit eines Wachmanns mit der einer Kellnerin oder einer Sekretärin im Büro?

Wie schon in den letzten Jahrzehnten werden auch in den kommenden die sekundären Dienstleistungen am stärksten expandieren und ihr Gewicht in der Erwerbstätigenstruktur deutlich erhöhen (+3,6 Prozentpunkte bis 2025). Im Vergleich mit den primären Dienstleistungen ist der Großteil der Berufe in den fünf Berufshauptfeldern dieses Bereichs durch anspruchsvolle Tätigkeiten gekennzeichnet. Sie sind in der Geschichte der Beschäftigung der Bereich, der bis ins letzte Drittel des zwanzigsten Jahrhunderts den überwiegenden Teil der Expansion der akademischen Berufe getragen hat (Riese 1967) und auch heute noch den größten Teil der Hochschulabsolventen absorbiert. Bereits 2005 stellte er knapp ein Drittel der Erwerbstätigen. Funktional ist er mit den technisch-naturwissenschaftlichen (2005 8,4 \% Anteil an den Erwerbstätigen), den Gesundheits- und Sozialberufen (11,2 \%), den Lehrberufen (3,7\%), den künstlerischen, Medien-, Geistes- und sozialwis- senschaftlichen Berufen (3\%) sowie den Rechts-, Managements- und wirtschaftswissenschaftlichen Berufen (4,6 \%) zum einen Teil auf Aufrechterhaltung und Weiterentwicklung der Produktion bezogen, zum anderen auf die Gewährleistung der sozialen und politischen Infrastruktur moderner Wohlfahrtsstaaten ausgerichtet. Dieser funktionalen entspricht - stärker als bei dem Gros der primären Dienstleistungen - eine institutionelle Differenzierung in teils privatwirtschaftliche, teils staatliche, teils auch selbstständig organisierte Erwerbstätigkeit.

Die Qualifikationsstruktur der Dienstleistungstätigkeiten im Sinne des in ihnen geforderten beruflichen Anforderungsniveaus ${ }^{2}$ lässt sich in Anlehnung an Tie-

2 Die Daten beruhen auf der BIBB/BAuA-Erwerbstätigenbefragung 2006, Kernerwerbstätige. Das Anforderungsniveau wurde mit Hilfe eines „subjektiven Ansatzes" ermittelt, der in der Qualifikationsforschung als besonders valide und zuverlässig gilt. Gefragt wurde, ,welche Art von Ausbildung für die Ausübung der Tätigkeit in der Regel erforderlich ist“ (Tiemann et al. 2008, S. 23). Die Antworten wurden anhand weiterer qualifikationsrelevanter Merkmale überprüft. 


\section{Abb. 2: Qualifikationsstruktur der Dienstleistungsberufsfelder}

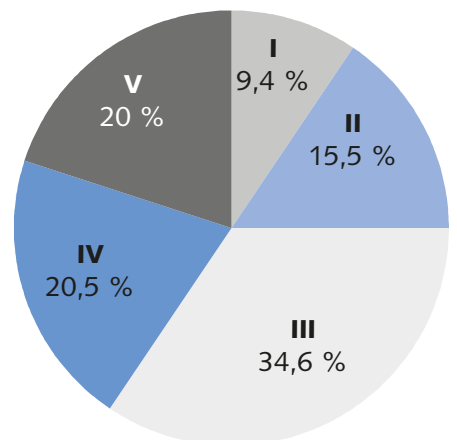

I: gering Qualifizierte (ca. $50 \%$ ohne Abschluss) z.B. Versandfertigmacher, Warenprüfer, Packer, Lagerarbeiter, Transportarbeiter, Reinigungs- und Entsorgungsberufe

II: mehrheitlich mit Berufsabschluss (über $30 \%$ ohne Abschluss) z.B. Verkaufs-, Verkehrs-, Bürohilfs-, Hotel-, Gaststättenberufe

III: dominant Qualifizierte (über 50 \% mit Berufsabschluss / unter $30 \%$ ohne Abschluss) z.B. Technische Fachkräfte, Bank-, Versicherungs-, Groß- u. Einzelhandelsbereich, kfm. Büroberufe, Gesundheitsberufe ohne Approbation

IV: gemischt Qualifizierte (50\% mit Berufsabschluss / über $25 \%$ Hochqualifizierte) z.B. Verwaltungsberufe ÖD, Finanz-, Rechnungswesen, IT-Kernberufe, Design-, Werbeberufe, Soziale Berufe

V: Hochqualifizierte (über $50 \%$, z. T. bis $90 \%$ HQA) z.B. MINT-Berufe, Rechts-, Management-, Lehrberufe, Gesundheitsberufe mit Approbation

mann et al. (2008) in fünf Niveaustufen entsprechend der Zusammensetzung der Erwerbstätigen des jeweiligen Dienstleistungsberufsfelds nach Beschäftigten ohne Berufsabschluss, mit abgeschlossener Berufsausbildung (inklusive Fachschulabschluss) und mit akademischem Abschluss darstellen (Abbildung 2):

Die Zuordnung der Dienstleistungsberufsfelder zu diesen fünf Clustern nach Niveaustufen der Qualifikationsanforderungen zeigt, dass die beiden niedrigsten Niveaustufen nur acht Berufsfelder umfassen, die alle zu den primären Dienstleistungen gehören. Mit den Hotel- und
Gaststätten- sowie den Reinigungsberufen sind in ihnen quantitativ größere Tätigkeitsbereiche vertreten, die in Zukunft sogar expandieren dürften (Abbildung 1). Für beide Niveaustufen gilt, dass die Anteile von Beschäftigten mit Hochschulabschluss minimal sind (zumeist unter $1 \%$ wie $\mathrm{Ta}$ belle 1 zeigt). Den Schwerpunkt bilden die Berufsfelder mit mittlerer Qualifikation (Kategorie 3), in dem der Hauptteil der primären Dienstleistungstätigkeiten untergebracht ist. Demgegenüber gehören fast alle Berufsfelder der beiden höchsten Kategorien (4 und 5) dem Bereich der sekundären Dienstleistungstätigkeiten an.

\section{Abb. 3: Qualifikationsniveaus der Dienstleistungsbeschäftigten - in $\%-$}

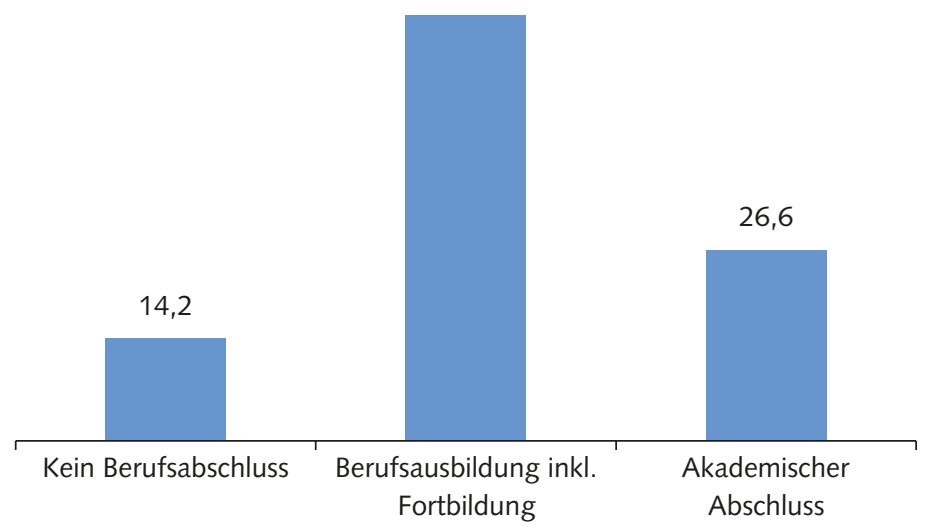

WSI MITTEILUNGEN
Betrachtet man die Gesamtheit der Dienstleistungsbeschäftigten über alle Berufsfelder hinweg nach den drei Niveaus der Qualifikationsanforderungen (ohne Berufsabschluss, Berufsausbildung, Akademischer Abschluss), so zeigt sich, dass die überwiegende Mehrheit auf dem Niveau eines mittleren Ausbildungsabschlusses (ca. $60 \%$ ), über ein Viertel auf akademischem Niveau und nur noch knapp ein Sechstel auf Ungelernten-Niveau tätig ist (Abbildung 3). ${ }^{3}$

Mit Blick auf die Entwicklungstendenzen der Dienstleistungstätigkeiten lässt sich die qualifikatorische Segmentation so interpretieren, dass die Hauptexpansion bei den beiden obersten Segmenten liegen wird. Hieraus begründet sich auch die aktuelle Diskussion über einen Mangel an Hochqualifizierten und die Notwendigkeit zur Erhöhung der Studierendenquote. Die im Durchschnitt sehr niedrigen Anteile von Akademikern auch im dritten Segment zeigt die Spaltung zwischen den Qualifikationsstufen. ${ }^{4}$ Allerdings bleibt auch die Polarisierung in der Qualifikationsstruktur der Dienstleistungsberufe erhalten. Auch wenn die Tendenz zu höher qualifizierten Tätigkeiten unübersehbar ist, verschwinden die gering qualifizierten Tätigkeiten nicht von der Bildfläche, sondern werden - ceteris paribus - besonders unter Einbezug von geringfügig Beschäftigten bei den Hotel- und Gaststättenberufen, im Einzelhandel, bei Lager- und Transportarbeiten sowie bei Bürohilfs- und bei Wachberufen Gewicht behalten. Freilich bleibt offen, ob man tatsächlich von einer Polarisierung oder eher von einer Dreiteilung sprechen sollte. Dies entscheidet sich daran, ob man das mittlere Segment zu dem höher qualifizierten rechnet oder als eine relativ stabile Mittelposition versteht.

3 Da es sich um eine subjektive Einschätzung handelt, wird man mit Blick auf die mittlere Kategorie trotz aller methodischen Kontrollen eine leichte Überhöhung der Qualifizierten anzunehmen haben. Eine weitere Einschränkung für die Validität der Verteilung muss man m.E. wegen der Konzentration der Befragung auf "Kernerwerbstätige $^{\prime \prime}$ (vgl. Fn. 2) machen müssen. Bei geringfügig Beschäftigten und Aushilfskräften, deren Zahl bei den primären Dienstleistungen beträchtlich groß ist, dürfte es sich vielfach um Tätigkeiten auf dem untersten Qualifikationsniveau handeln.

4 Die Differenzierungen sind zu einem Gutteil der Klassifikation der Berufe geschuldet, in die das Qualifikationsniveau der Berufstätigkeiten mit eingegangen ist. 


\begin{tabular}{|c|c|c|c|c|}
\hline \multirow[t]{2}{*}{ Berufsfelder } & \multirow[b]{2}{*}{ Insgesamt } & \multicolumn{3}{|c|}{ Qualifikationsniveau 2006} \\
\hline & & Kein Berufsabschluss & $\begin{array}{l}\text { Berufsausbildung inkl. } \\
\text { Fortbildung }\end{array}$ & $\begin{array}{l}\text { Akademischer } \\
\text { Abschluss }\end{array}$ \\
\hline & Anzahl & \multicolumn{3}{|c|}{- in $\%-$} \\
\hline Warenprüfer/-innen, Versandfertigmacher/innen & 549.437 & 50,1 & 48,7 & 1,3 \\
\hline Reinigungs-, Entsorgungsberufe & 859.471 & 72,8 & 27,1 & 0,1 \\
\hline Packer/-innen, Lager-, Transportarbeiter/-innen & 948.334 & 48,5 & 50,7 & 0,8 \\
\hline Verkaufsberufe (Einzelhandel) & 1.572 .475 & 31,9 & 66,5 & 1,6 \\
\hline Verkehrsberufe & 1.052 .418 & 36,6 & 62,8 & 0,6 \\
\hline Bürohilfsberufe, Telefonisten und Telefonistinnen & 412.728 & 37,4 & 56,2 & 6,4 \\
\hline Personenschutz-, Wachberufe & 184.467 & 33,2 & 65,9 & 1,0 \\
\hline Hotel-, Gaststättenberufe, Hauswirtschaft & 650.864 & 39,3 & 59,4 & 1,3 \\
\hline Techniker/-innen & 1.023 .884 & 1,6 & 89,8 & 8,5 \\
\hline Technische Zeichner/, verwandte Berufe & 62.692 & 0,0 & 99,2 & 0,8 \\
\hline Vermessungswesen & 56.718 & 4,6 & 76,1 & 19,4 \\
\hline Technische Sonderkäfte & 120.973 & 5,1 & 90,7 & 4,2 \\
\hline Groß-, Einzelhandelskaufleute & 895.573 & 6,4 & 85,1 & 8,5 \\
\hline Bank-, Versicherungsfachleute & 922.180 & 1,1 & 84,3 & 14,6 \\
\hline Sonstige kaufmännische Berufe (ohne Groß-, Einzelhandel, Kreditgewerbe) & 830.995 & 7,9 & 78,7 & 13,3 \\
\hline Luft-, Schifffahrtsberufe & $(52.390)$ & $(13,7)$ & $(67,2)$ & $(19,2)$ \\
\hline Kaufmännische Büroberufe & 2.040 .992 & 9,3 & 85,0 & 5,7 \\
\hline Hausmeister/-innen & 253.756 & 25,2 & 74,8 & 0,0 \\
\hline Gesundheitsberufe ohne Approbation & 2.188 .291 & 5,2 & 88,3 & 6,5 \\
\hline Berufe in der Körperpflege & 190.290 & 2,2 & 97,8 & 0,0 \\
\hline Werbefachleute & 256.978 & 5,7 & 48,3 & 45,9 \\
\hline Verwaltungsberufe im ÖD & 1.439 .836 & 2,2 & 62,1 & 35,7 \\
\hline Finanz-, Rechnungswesen, Buchhaltung & 695.073 & 4,7 & 70,8 & 24,5 \\
\hline IT-Kernberufe & 818.677 & 1,2 & 47,8 & 51,0 \\
\hline Sicherheitsberufe & 527.761 & 2,6 & 61,5 & 35,9 \\
\hline Künsterler/-innen, Musiker/-innen & 127.573 & 14,7 & 47,4 & 37,9 \\
\hline Designer/-innen, Fotografen/-innen, Reklamehersteller/-innen & 138.540 & 6,9 & 54,3 & 38,7 \\
\hline Soziale Berufe & 1.122 .611 & 6,5 & 50,1 & 43,3 \\
\hline Ingenieure und Ingenieurinnen & 1.040 .008 & 0,4 & 9,2 & 90,3 \\
\hline Chemiker/-innen, Physiker/-innen, Naturwissenschaftler/-innen & 137.513 & 2,5 & 9,5 & 88,0 \\
\hline Geschäftsführung, Wirtschaftsprüfung, Unternehmensberatung & 1.205 .805 & 1,1 & 44,1 & 54,7 \\
\hline Rechtsberufe & 238.488 & 0,0 & 20,3 & 79,7 \\
\hline Gesundheitsberufe mit Approbation & 332.248 & 0,3 & 8,8 & 90,9 \\
\hline Lehrer/-innen & 1.662 .457 & 1,7 & 13,1 & 85,2 \\
\hline Publ., Bibliotheks-, Übersetzungs-, verwaltende Wissenschaftsberufe & 389.537 & 10,4 & 28,5 & 61,1 \\
\hline Insgesamt (der ausgewählten Berufsfelder) & 25.002 .033 & 14,2 & 59,2 & 26,6 \\
\hline
\end{tabular}

\section{3 \\ Arbeitstypus und Kompetenz- profile von Dienstleistungs- tätigkeiten}

Die bisherige Darstellung hat die funktionale Heterogenität der Dienstleistungstätigkeiten, die heute ca. 80 \% der Erwerbstätigkeit in der Bundesrepublik ausmacht, und deren Qualifikationsstruktur in Form von Ausbildungsniveaus beschrieben. Dabei sind zwei Fragen offen geblieben: zum einen, ob es in der Vielzahl der Tätigkeiten so etwas wie einen gemeinsamen Kern gibt, einen Arbeitstypus, der Relevanz für die Organisation der Arbeit hat, ähnlich wie es - jenseits des Lohnarbeitsverhältnisses - die Auseinandersetzung mit Gegenständen und Maschinen für industrielle Herstellungsarbeit hatte und hat; zum anderen, welche Kompetenzen auf dem jeweiligen Ausbildungsniveau zur Bewältigung der alltäglichen Arbeit erforderlich sind. Bei beiden Fragen kann es nicht um die Aufzählung eines Katalogs von einzelnen Arbeitsformen oder inhaltlichen Kompetenzen gehen, sondern um die Suche nach so etwas wie gemeinsamen Kernen in Arbeits- und Kompetenzprofilen, die zwar fachspezifische Einfärbungen haben können, aber zugleich berufsübergreifende Gültigkeit beanspruchen können.

Für beide Fragen soll im Folgenden ein Diskussionsvorschlag vorgetragen werden, für den klar sein sollte, dass er nicht für die Gesamtheit, aber - im Sinne eines Idealty- pus - für große Teile der Dienstleistungsberufe, inzwischen wohl auch bei vielen industriellen Tätigkeiten, relevant ist.

\subsection{NEUER ARBEITSTYPUS: DIENSTLEISTUNGSTÄTIGKEIT ALS INTERAKTIVE ARBEIT}

Wie man als das Gemeinsame von inhaltlich unterschiedlichen Industrietätigkeiten die technisierte Auseinandersetzung mit Gegenständen verstehen kann, so lässt sich Interaktivität als Gemeinsames von Dienstleistungstätigkeiten im Sinne eines neuen, in der Erwerbsstruktur dominanten Arbeitstypus begreifen.

Wo immer sie auch ausgeübt werden: Fast überall sind Dienstleistungstätigkeiten interaktive Arbeit. Das heißt, eine 
Arbeit, die unmittelbar bedürfnisbezogen auf ein konkretes Gegenüber gerichtet ist, dessen Wille die Richtschnur für das Arbeitshandeln abgibt (bzw. abgeben sollte $)^{5}$, selbst wenn der Wille oder das Bedürfnis nicht in präzisen Anweisungen artikuliert werden kann. Das Bedürfnis des Gegenüber - handele es sich um einen Kunden im Warenaustausch, um einen Klienten im Beratungs- oder Betreuungsgeschäft oder einen Patienten im Pflegeund Gesundheitswesen - zu präzisieren und gemeinsam Wege zu seiner Befriedigung zu erarbeiten, macht den Kern der Interaktivität von Dienstleistungsarbeit aus. Das Gegenüber ist nicht nur Adressat, sondern zugleich Mitproduzent der Tätigkeit (vgl. schon früh Badura/Gross 1976; Gross 1983); hierin liegt ein fundamentaler Unterschied zur Industriearbeit, in der Interaktion bzw. Kommunikation Mittel zum Zweck war, während sie hier wesentlicher Inhalt ist. Dieser Typ von Arbeit findet sich am stärksten ausgeprägt in allen personenbezogenen sozialen und Gesundheitsdienstleistungen, weiter in allen Beratungs- und Kommunikationsdienstleistungen von Banken und Versicherungen bis zur Arbeitsverwaltung und zu Call-Centern (vgl. Baethge 2011). ${ }^{6}$

Eine solche, vom unmittelbaren Arbeitsprozess und Tätigkeitsinhalt ausgehende Definition kann im Sinne der Unterscheidung von Kohn (1977) zwischen „Umgang mit Sachen“, „Umgang mit Personen“ und „Umgang mit Symbolen“ eine Differenz zwischen industrieller (gegenstandsbezogener) und Dienstleistungsarbeit (personenbezogen und wissens- bzw. symbolbezogen) sichtbar machen. Zur Analyse der unterschiedlichen Formen und Probleme von Dienstleistungsarbeit greift eine solche Definition aber zu kurz, da die systemischen Dimensionen der Arbeit in dieser nur arbeitsprozesslichen Definition, die gleichwohl den Ausgangspunkt bilden muss, ausgeblendet sind. Wie alle Erwerbsarbeit aber ist auch Dienstleistungsarbeit in die institutionellen Ordnungen eingebunden, in denen sich die Wirtschaft einer Gesellschaft bewegt, ob sie beispielsweise mehr privater Kapitalverwertung dient und auf Wettbewerbsmärkten operiert oder sich mehr in politisch definierten, idealiter am Gemeinwohl orientierten Institutionen bewegt.

Ob man interaktive Arbeit eng prozessual oder systemisch mit Blick auf die Formbestimmtheit der Arbeit definiert, ist für die Analyse von Dienstleistungstätigkeiten folgenreich. Nicht nur im pädagogischen und therapeutischen Bereich liegt es nahe, Dienstleistungsarbeit im Rahmen interaktionstheoretischer Konzepte zu analysieren. In dem Teil der Arbeitssoziologie, die sich in der Tradition subjektorientierter Soziologie versteht, wird Interaktivität von Arbeit geradezu zu einem neuen arbeitssoziologischen Paradigma stilisiert. ${ }^{7}$ Dienstleitungen werden hier als Arbeit bezeichnet, die sich dadurch auszeichnet, „dass Beschäftigte im Arbeitsprozess, Experten und Klienten, Dienstleistungsbeschäftigte und Kunden aktiv zusammenarbeiten müssen, damit die Arbeitsaufgabe erfüllt oder die Dienstleistungen erbracht werden kann" (Dunkel/Weihrich 2010, S. 177).

Es mag verführerisch sein, diesen neuen Charakter von Interaktion in der Arbeit zum Wesentlichen der Analyse von Dienstleistungstätigkeiten $\mathrm{zu}$ machen. Dies allerdings wäre nur dann gerechtfertigt, wenn der Nachweis gelänge, dass die Interaktion in den Arbeitsprozessen die institutionellen Formen, in denen sie sich vollzieht, ausschalten und tatsächlich als herrschaftsfreie Interaktion zwischen Dienstleister und Kunde/Klient/Patient ablaufen kann. Dieser Nachweis gelingt den von der subjektorientierten Soziologie kommenden Autoren bisher nicht, sie versuchen nicht einmal, ihn anzutreten. Der einfache Hinweis darauf, dass interaktive Arbeit „durch die betriebliche Herrschaft nur begrenzt organisierbar“ sei (ebd.), artikuliert zunächst einmal nur das Kontroll-Dilemma, in dem sich Unternehmen gegenüber jenen Beschäftigten befinden, die diesen Typ von Arbeit ausführen. Er besagt nicht, dass die betriebliche Herrschaft die Arbeiten nicht doch so weit wie möglich steuert und kontrolliert. Die Geschichte der Angestelltensoziologie handelt fast von nichts anderem als von diesem Kontroll-Dilemma und den Versuchen der Kapitaleigner, es durch „weiche“ Formen der Steuerung und Kontrolle in den Griff zu bekommen. Erst die Verbindung von (arbeits)prozessualen Aspekten der Interaktivität und systemischen Merkmalen ihrer institutionellen Formbestimmtheit verspricht eine angemessene wissenschaftliche Analyse von Dienstleitungsarbeit.

Die Doppelperspektive von Arbeitsprozess (Interaktivität) und systemischer Einbindung generiert ein permanentes Spannungsverhältnis, das auf die alltägliche Arbeitsgestaltung einwirkt. Dass
Interaktivität nur begrenzt technisierbar, rationalisierbar und standardisierbar ist und deswegen für die economies of scale Barrieren in der Marktverfassung eines nicht anonymen Marktes liegen, hindert nicht, dass von den Dienstleistungsunternehmen - übrigens privaten wie öffentlichen - Anstrengungen unternommen werden, die arbeitsprozesslich gesetzten Begrenzungen in allen Dimensionen (Technisierung, Standardisierung, Rationalisierung) zu durchbrechen. Hieraus resultieren nicht allein Arbeitskonflikte, sondern auch Auseinandersetzungen über die Qualität von Dienstleistungen.

Um einem Missverständnis vorzubeugen: Grenzen von Technisierbarkeit und Standardisierung heißt nicht, dass Technik und Standardisierung in einem konstitutiven Gegensatz zum Ziel von Dienstleistungen stünden. Es gibt in der jüngeren Dienstleistungsgeschichte eine Fülle von Beispielen, wo beide Strategien (Technisierung und Standardisierung) ineinandergreifen und hoch positive und produktive Effekte auf Dienstleistungsqualität und -versorgung hervorgebracht haben: von der Einführung des bargeldlosen Zahlungsverkehrs über elektronische Informationsverarbeitung und -vermittlung bis hin zu Hochtechnologie in der medizinischen Diagnose und Therapie. Allerdings gibt es für die gleichen Tätigkeitsbereiche auch genügend Beispiele für Ambivalenzen der Technisierung und Standardisierung, die zeigen, dass Technikeinsatz in Dienstleistungsfeldern, vor allem bei personenbezogenen Dienstleistungen, komplizierter als in der industriellen Produktion ist. Ist für Letztere Technik oft das Ziel und der Weg zur Herstellung neuer technischer Produkte, so bleibt sie für Dienstleistungen Mittel der individuellen Bedürfnisbefriedigung und ist unter deren Kriterien zu entwi-

5 Ein unmittelbarer Bedürfnisbezug als Definitionsmerkmal der Dienstleistungstätigkeit gilt nicht für viele der unternehmens- bzw. produktionsbezogenen Dienstleistungen wie z. B. viele IT-Dienste. Bei ihnen überwiegen oft die technischen Dimensionen der Arbeit deren kommunikative Einbindung. Deswegen ist es nicht verwunderlich, dass in ihnen oft die Analogie zur Industriearbeit die wissenschaftliche Analyse prägt.

6 Man kann darüber streiten, ob nicht selbst die beträchtliche Anzahl von back-office Tätigkeiten in den Verwaltungen großer Dienstleistungsunternehmen indirekt interaktive Arbeit darstellt, da sie Zuarbeit- und Nacharbeitstätigkeiten für unmittelbare Kundenkontakte verrichten.

7 So beispielsweise in dem von Böhle et al. (2010) herausgegebenen Handbuch Arbeitssoziologie. 
ckeln und einzusetzen. Je weiter entfernt von der unmittelbaren Bedürfnisbefriedigung Technik angesiedelt ist, desto weniger kompliziert ist ihre Anwendung. Umgekehrt gilt auch: Je unmittelbarer Technik den Dienstleistungsprozess beeinflusst, umso intensiver ist ihre Wirkung auf den Interaktionsprozess, der zur Bedürfnisbefriedigung führt, in ihren Anwendungsvoraussetzungen und -folgen zu reflektieren und in Gestaltungskonzepten von Dienstleistungsarbeit zu berücksichtigen. Blinde High-Tech-Euphorie kann schnell zu problematischen Effekten für Dienstleistungsqualität und Unternehmenserfolg führen. Auch hierfür gibt es genügend Beispiele (vgl. Breisig et al. 2010, S. 305ff.; Oberbeck 2001, S. 71ff.).

Die Auseinandersetzung um Rationalisierung, Technisierung und Standardisierung ist kein akademischer Schulen-Streit, sie hat einen harten praktischen Kern. Er bezieht sich auf Formen der Arbeitsteilung, auf Leistungs- und Pensumsdefinitionen für Dienstleistungsarbeit, auf Normierung der Arbeit, auf Leitbilder für Dienstleistungsqualität, auf Handlungsspielräume in der Arbeit und mögliche Konflikte zwischen Dienstleistungstätigen und Unternehmensmanagement. All diese Aspekte betreffen nicht allein die Arbeitssituation und die Qualität der Arbeit für die Beschäftigten, sie prägen immer zugleich auch die Qualität der Dienstleistung für Kunden und Klienten. Darin unterscheidet sich Dienstleistungsarbeit grundlegend von industrieller Herstellungsarbeit, in der ein solch enger Zusammenhang von Qualität der Arbeit und Qualität des Produkts nicht existiert. Die Konsequenzen dieser konstitutiven Besonderheit von interaktiver Dienstleistungsarbeit für Kompetenzprofile und Professionalisierung sind im Weiteren zu erörtern.

\subsection{ARBEITSLOGIK UND KOMPE- TENZPROFILE VON DIENSTLEIS- TUNGSTÄTIGKEITEN}

Die Betrachtung des Arbeitstypus interaktive Dienstleistungsarbeit lässt sich dahingehend resümieren, dass mit ihm im Vergleich zur Industriearbeit eine neue Arbeitslogik in der Erwerbsarbeit Fuß gefasst hat. ${ }^{8}$

Unterschiedliche Arbeitslogiken bedingen unterschiedliche Qualifikations- und Kompetenzprofile. Eine erste Annäherung an Kompetenzunterschiede lässt sich - in einem säkularen Betrachtungshorizont - über den Wandel der Wissensformen gewinnen. Ihn kann man mit Polanyi (1985) und Nonaka/Takeuchi (1997) als Tendenz von der Dominanz impliziten zur Vorherrschaft expliziten Wissens beschreiben. Implizites bzw. Erfahrungswissen ist dadurch charakterisiert, dass es in unmittelbaren Lebens- und Arbeitszusammenhängen angeeignet wird, an die Person und kontextgebunden, schwer übertragbar und verallgemeinerbar ist; handwerkliches Geschick und Wissen können als Inbegriff dieses Wissenstypus gelten. Demgegenüber umfasst explizites oder systematisches Wissen alle Formen kodifizierten Wissens, das in praxisenthobenen Einrichtungen (z.B. Schulen, Hochschulen, Forschungsstätten) generiert und vermittelt wird; sein systematischer Charakter und seine Kontextunverbundenheit machen es relativ leicht übertragbar. Es existiert kaum ein Entwicklungstheoretiker, der diesen Wissenstypus nicht zum zentralen Merkmal nachindustrieller Gesellschaft erklärt (allen voran Bell 1975).

Wie bei den Arbeitstypen handelt es sich auch bei den beiden Wissensformen um idealtypische Konstruktionen auf hohem Abstraktionsniveau, die in der Realität in vielfältigen Kombinationen auftauchen. In Arbeitszusammenhängen kommt heute Erfahrungswissen selten ohne systematisches Wissen als Grundlage für Erfahrungsverarbeitung aus. Ebenso wenig kann in praktischen Anwendungszusammenhängen systematisches/explizites Wissen auf Erfahrung verzichten. Entscheidend für Berufsbildung und Kompetenzprofile ist, welcher Wissenstypus in den jeweiligen Arbeitszusammenhängen dominiert. Im Folgenden soll die Entwicklung der Kompetenzprofile an den beiden für interaktive Arbeit zentralen Dimensionen des ,analytischen Wissens“ und der „sozialen Kommunikation" beleuchtet werden.

\section{ANALYTISCHES WISSEN}

Mit Blick auf die skizzierten Wissensformen gilt, dass die säkulare Tendenz vom impliziten zum expliziten Wissen für beide großen Sektoren der Volkswirtschaft, Industrie- und Dienstleistungssektor, Gültigkeit hat. Gemeinsam ist beiden, wenn vielleicht auch mit unterschiedlichem Gewicht, dass hohe kognitive Analysekompetenzen eine zunehmende Rolle spielen, als Voraussetzung dafür, im Arbeitsprozess überhaupt Erfahrungen machen und auch implizites Wissen, das zur Lösung konkreter Arbeitsaufgaben unverzichtbar bleibt, richtig einsetzen zu können.

In einer Studie des BIBB wird die hohe Bedeutung des expliziten Wissens anhand der Anforderungen an Methodenkompetenzen, die man als einen Kernbereich systematischen Wissens betrachten kann, deutlich. Bei den unterschiedlichen Methodenkompetenzen weisen die Produktionsberufe im Durchschnitt die niedrigsten, die sekundären Dienstleistungsberufe die höchsten Prozentsätze der Nennungen auf (Abbildung 4). Die Komplexität der analytischen Wissensanforderungen entsteht vor allem durch das Nebeneinander von Aufgabenvielfalt, schwierigen Entscheidungsprozessen und der Vermittlung schwieriger Sachverhalte. Man kann die Verteilungen in Abbildung 4 als Beleg dafür nehmen, dass Dienstleistungsberufe in der Regel höhere Wissenskompetenzen als Produktionstätigkeiten verlangen, dass sich aber auch in diesen die zunehmende Wissensbasierung der Arbeitsabläufe durchzusetzen scheint.

\section{SOZIALE KOMMUNIKATION}

Wie schon betont, wird Kommunikationsfähigkeit als fachübergreifende Kompetenz bei interaktiver Arbeit die Basiskompetenz. Sie bezieht sich damit nicht wie in der industriellen Arbeit vorrangig auf die Kooperation mit Kollegen und Vorgesetzten in der gemeinsamen Bearbeitung eines Gegenstands oder einer Aufgabe (dieser Typ von Kommunikation spielt selbstverständlich auch eine Rolle). Das Neue ist, dass Kommunikation sich auf Kunden/ Klienten/Patienten als Ko-Produzenten der Dienstleistung richtet und in vielen Feldern den funktionalen Kern der Dienstleistung darstellt, weil Erstellung und Konsumtion der Leistung in einem (kommunikativen) Arbeitsakt zusammenfällt.

Das unterschiedliche Gewicht, das Kommunikationsfähigkeit in Industrieund Dienstleistungsfachkräftetätigkeiten hat, wird durch die repräsentative Studie

8 Es versteht sich von selbst, dass man den idealtypischen Gegensatz zur Industriearbeit nicht überstrapazieren darf. Im Zuge der internen Tertiarisierung industrieller Produktion erfährt die hier skizzierte Arbeitslogik zunehmend auch Eingang in die Produktion. Zudem bleibt im Auge zu behalten, dass die Kategorie der Arbeitslogik in den unterschiedlichen Dienstleistungsfeldern unterschiedliche Ausprägungen aufweist. 
von Hall et al. (2007) an vier Dimensionen sozialer Kompetenz eindrucksvoll ausgewiesen. In allen vier Dimensionen („Andere überzeugen und Kompromisse aushandeln; Freie Reden oder Vorträge halten; Kontakte zu Kunden, Klienten oder Patienten; Besondere Verantwortung für andere Menschen) zeigt sich eine klare Stufung von den Produktionsberufen zu den primären und sekundären Dienstleistungsberufen (Abbildung 5). Die mit Abstand höchsten Werte erreichen die sekundären Dienstleistungsberufe, bei denen die
Sozial- und Gesundheitsdienstberufe im mittleren Fachkräftesektor zahlenmäßig dominieren.

Im internationalen Vergleich zeigen sich analoge Tendenzen in den Kompetenzprofilen wie in Deutschland. Sowohl in den kaufmännischen (business and administration) als auch in den sozialen und pflegerischen Dienstleistungsberufen (social and health care) erhalten in allen acht in eine internationale Vergleichsstudie einbezogenen Ländern Wissensqualifikationen im Sinne analytischer Fähig-

\section{Abb. 4: Anforderungen an Methodenkompetenzen nach Berufssektoren - in \% -}

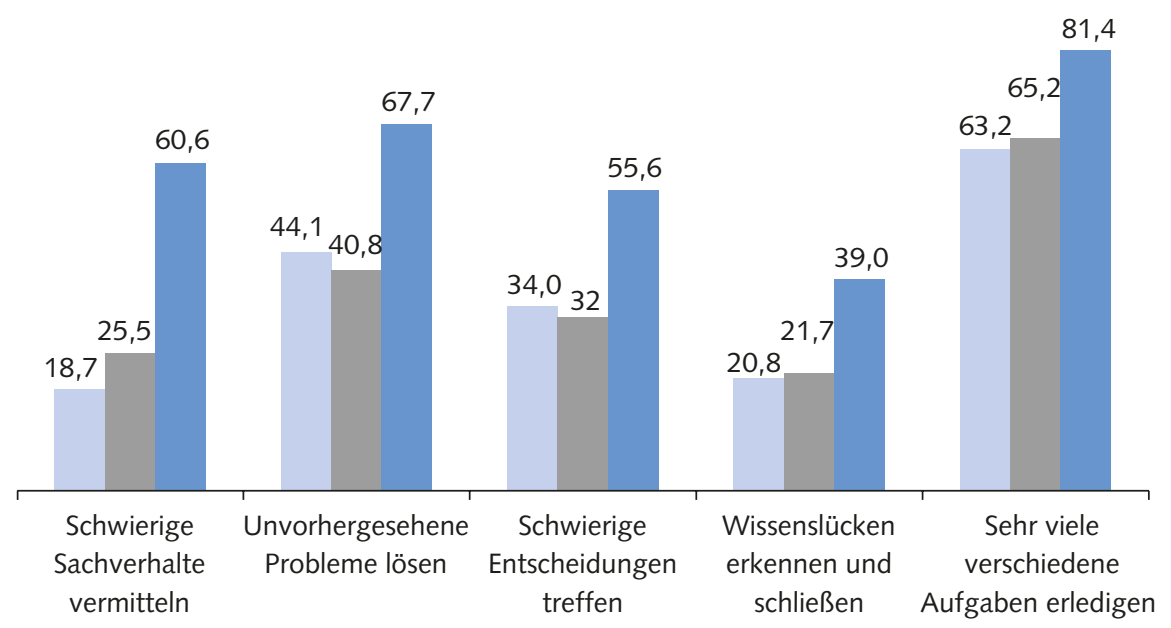

Produktionsberufe $\square$ Primäre Dienstleistungsberufe $\square$ Sekundäre Dienstleistungsberufe

\section{Abb. 5: Anforderungen an Sozialkompetenzen nach \\ Berufssektoren - in \% -}

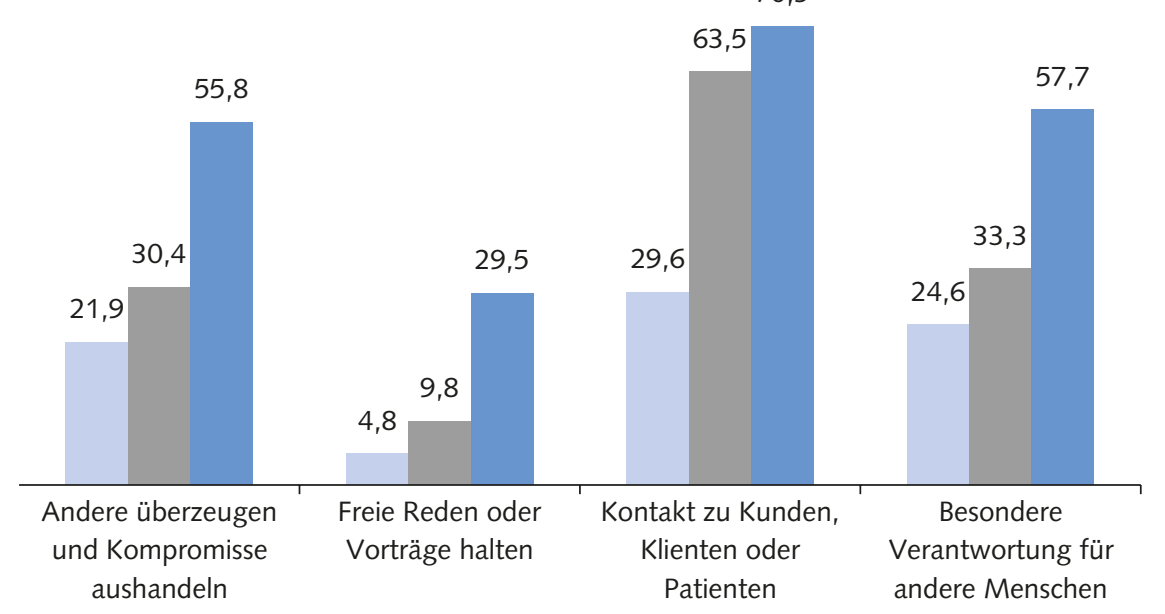

Produktionsberufe $\square$ Primäre Dienstleistungsberufe $\square$ Sekundäre Dienstleistungsberufe keiten zur Beurteilung von Situationen und Sachverhalten und Denken in Zusammenhängen zur Lösung von Problemen auf der einen, soziale Kompetenzen der inter- und intradisziplinären Kooperation sowie die Fähigkeit, selbstständig Beziehungen aufzubauen und zu gestalten, die höchsten Bewertungen der Experten (vgl. Baethge/Arends 2009, S. 85ff.). Man darf sich allerdings weder die sozialen noch die kognitiven Kompetenzen als von fachlichen Inhalten losgelöste Metakompetenzen vorstellen, sie verbinden sich in der Regel mit berufsfachlichen Qualifikationen, die ihnen ihre spezifische Ausprägung verleihen.

Für die Berufsausbildung heißt das, dass sie nicht nach dem traditionellen Muster der en-passant-Lehre, für die das Prinzip "Zuschauen und Nachahmen“ steht, organisiert werden kann. Es bedarf, damit arbeitsintegrierte Ausbildungssequenzen greifen können, wissensbezogener Sequenzen der Vorbereitung und Begleitung. Die kognitiv-reflexiven Kompetenzen spielen gerade bei personenbezogenen Dienstleistungen - von jedweden Beratungs- bis hin zu Pflegetätigkeiten - immer wieder eine wichtige Rolle. Es könnte der hier skizzierte Zusammenhang von kommunikativen und systematischen Wissenskompetenzen sein, der die Unternehmen selbst für die Rekrutierung ihrer Auszubildenden und Fachkräfte auf der mittleren Ebene zunehmend auf Absolventen mit höheren Bildungsabschlüssen zurückgreifen lässt, was in den letzten beiden Jahrzehnten zu einer stabilen Segmentation in der dualen Berufsausbildung nach schulischem Vorbildungsniveau geführt hat (vgl. Baethge 2010).

\section{1 \\ Professionalisierung und Professionalität}

Tätigkeitsstrukturen und Kompetenzprofile der beschriebenen Art erfordern andere Professionalisierungspfade, als sie für Entstehungsphase und vor allem Blütezeit der Industrialisierung mit der Etablierung des Berufstypus Facharbeiter und der ihm entsprechenden dualen Facharbeiterausbildung tonangebend und erfolgreich waren. Da die verstärkte Wissensbasierung auch vor den Produktionsberufen nicht halt gemacht hat und es auch zu deren zu- 
nehmender internen Tertiarisierung ${ }^{9}$ gekommen ist, gelten die folgenden Überlegungen bis zu einem gewissen Grade auch für Produktionsberufe.

Das erste Merkmal betrifft die durchgängige Anhebung der kognitiven Voraussetzungen in der Berufsausbildung, die man der Dominanz des systematischen Wissens zuschreiben muss. Sie äußert sich darin, dass in den letzten Jahrzehnten in den anspruchsvolleren Produktions- und Dienstleistungsberufen fast nur noch Abiturienten oder Realschulabsolventen in die Ausbildung aufgenommen werden und hier auch verstärkte Weiterbildungsanstrengungen in den Unternehmen zu beobachten sind (vgl. Autorengruppe Bildungsberichterstattung 2008, S. 285; Baethge-Kinsky et al. 2006). Die weiteren Merkmale wurzeln in dem starken Gewicht der sozialen und reflexiven Kompetenzanforderungen, die für alle interaktiven (Dienstleistungs-)Arbeiten konstitutiv sind. Sie verweisen auf Fähigkeiten und Dispositionen selbstständiger Analyseund Urteilsfähigkeit sowie Reflexivität über die eigene Rolle in komplexen Arbeitssituationen, darüber hinaus auf kaum in Ausbildung vermittelbare Dispositionen, die in der neueren Organisationspsychologie mit „psychological capital“ in die Diskussion gebracht worden sind und $\mathrm{zu}$ denen Selbstwirksamkeitsüberzeugungen, Optimismus und Zähigkeit bei der Verfolgung von Zielen zählen (vgl. Avey et al. 2010). Diese allgemeinen und berufsübergreifenden Kompetenzen sind gleichwohl nicht völlig losgelöst von den beruflichen Kontexten, in denen sie wirksam werden, und verbinden sich deswegen in der Regel mit berufsfachlichen Fähigkeiten.

Es ist diese Verlagerung in den Arbeitsanforderungen zu inneren Dispositionen, die in der Berufsbildungstheorie - zum Teil auch in der -praxis - der Kompetenzkategorie ein zunehmend höheres Gewicht verschafft hat. ${ }^{10}$ Qualifikation als Kategorie für extern gesetzte Arbeitsanforderungen und Kompetenz als individuelle Performanz-Kategorie ${ }^{11}$ rücken in dem Maße näher aneinander, in dem die Anforderungen immer mehr in situativen Leistungen der Wahrnehmung und Interpretation von Problemen bestehen. Solche Leistungen basieren auf kognitiven Fähigkeiten und Verhaltensdispositionen, die in immer wieder neuen Interaktionssituationen aktiviert werden müssen. Wie gezeigt, sind die Kompetenzen unterschiedlich dimensioniert. Neben den analytischen Wissens- und den sozialen Kommunikationskompetenzen sollten Kompetenzen des Umgangs mit IuK-Technik nicht vernachlässigt werden. Das Problem für die Berufsausbildung liegt darin, dass alle drei genannten Kompetenzdimensionen immer fachspezifische Ausprägungen haben. Was der Bedeutungszuwachs der Kompetenzkategorie für die Ausbildungsorganisation bedeutet, ist schwer zu sagen. Er präjudiziert nicht von sich aus eine bestimmte-z.B. schulische oder betriebliche - Ausbildungsform. Aber jedwedes Ausbildungsarrangement ist daran zu messen, wieweit es ihm gelingt, diese Kompetenzen zu vermitteln.

Der Wechsel in den Kompetenzprofilen kann nicht folgendlos für die berufliche Identität, die zweite Säule der Berufskategorie, bleiben. Berufliche Identität ist in ihrer doppelten Perspektive als individuelle Motivations- und als soziale Anerkennungsform für berufliches Handeln von zentraler Bedeutung. Bei Industriearbeit wurde die berufliche Identität der Facharbeiter wesentlich im Begriff des „Produzentenstolzes“ gefasst. Wie es der Begriff ausdrückt, ist Produzentenstolz eine Kategorie industrieller Herstellungsarbeit, in der die soziale Anerkennung und das individuelle Selbstwertgefühl stark von der Gegenständlichkeit und Anschaulichkeit eines funktionstüchtigen Produkts sowie der Kollektivität der Arbeitsprozesse abgeleitet waren.

Diese Kategorie auf Dienstleistungstätigkeiten anzuwenden, wie es in der jüngeren Dienstleistungsdebatte immer wieder vorgeschlagen wird, verweist insofern in die falsche Richtung, als ihre spezifischen Konstitutionsbedingungen in kollektiven Herstellungsprozessen und allgemeiner Wahrnehmbarkeit der Produkte ausgeblendet werden. Nicht, dass Berufsstolz nicht auch für Dienstleistungsarbeit ein zentrales Moment der individuellen und sozialen Identität und eine wichtige Grundlage für die Qualität der Arbeit bildete. Aber Berufsstolz muss in interaktiver Dienstleistungsarbeit anders in einer Weise definiert werden, die dem individualisierten Charakter der Tätigkeit und ihrer nach außen oft nicht breit wahrnehmbaren Ergebnisse Rechnung trägt.

Berufliche Identität bei Dienstleistungstätigkeiten wurzelt mehr in den individuellen Kompetenzen und inneren Verhaltensdispositionen als in außen sichtbaren Attributen, bedarf aber der externen Anerkennung. Insofern steht sie dem Professionsbewusstsein der traditionellen professions (Ärzte, Rechtsanwälte u. a.) näher als dem Facharbeiterbewusstsein. Ein solches Kompetenzbewusstsein zu entwickeln, ist aber kein nur reflexiver Prozess des jeweiligen Kompetenzträgers, es muss fundiert sein in einem eigenständigen Professionalisierungsprozess. Dieser basiert auf einer Fachausbildung und auf gesellschaftlichen Anerkennungsverhältnissen auf unterschiedlichen Ebenen: auf der politischen Ebene durch Setzung von Qualitätsstandards für berufliches Handeln in Institutionen; ${ }^{12}$ auf dem Arbeitsmarkt durch angemessene Entlohnung. Entscheidend aber erscheint die Ebene der betrieblichen Arbeitsorganisation (gleichgültig, ob in privaten oder öffentlichen Betrieben). Alle politischen oder gesellschaftlichen Regulationen (z.B. durch Fachverbände) bleiben deklamativ, wenn nicht in der betrieblichen Arbeitsorganisation die Kompetenz der Dienstleister Anerkennung findet: durch klare Verantwortungszuweisungen, die Dienstleistungstätigen Selbstständigkeit bei der Durchführung ihrer Arbeit und Handlungsspielräume ebenso sichern wie eine eigene Position gegenüber Vorgesetzten; ferner durch sich selbst organisierende Gruppenarbeit und andere lernförderliche und Kompetenz erhaltende Arbeitsformen sowie die Einräumung beruflicher Entwicklungsperspektiven.

Wenn man die Qualität von Dienstleistungen in einer volkswirtschaftlichen Perspektive an der bestmöglichen Be-

9 Mit "interner Tertiarisierung “ industrieller Produktion wird der Prozess bezeichnet, der sich in einem zunehmenden Ausbau der indirekten Bereiche in Produktionsunternehmen ausdrückt und den Ausbau der Wissens- und marktbezogenen Abteilungen und eine verstärkte Markt- und Kundenorientierung in den Produktionsabteilungen zum Inhalt hat.

10 Über zehn Jahre Arbeit im Rahmen der "Arbeitsgemeinschaft betrieblicher Weiterbildungsforschung "legen Zeugnis von diesem Wandel ab, der in zehn Jahrbüchern "Kompetenzentwicklung “ von 1996-2005 seinen literarischen Niederschlag gefunden hat (vgl. Arbeitsgemeinschaft QUEM (Hrsg.) (verschiedene Jahrgänge)

11 Es existiert bis heute kein einheitliches Verständnis von Kompetenz in der Wissenschaft. Für die Vielfalt der Kompetenz-Konzepte vgl. Baethge et al. (2006), S. 13ff.

12 In den letzten Jahren sind einzelne Ansätze dazu im Sinne von Kundenschutzrechten beispielsweise für Pflegeheime, in jüngster Zeit selbst für Finanzdienstleistungen in Deutschland zu beobachten. 
friedigung von Kunden-/Klienten-/Patientenbedürfnissen misst, kann man die Rolle professioneller Identität, die auch ein Berufsethos einschließt, für die Qualität von Dienstleistungen nicht hoch genug einschätzen. Alle politischen und gesellschaftlichen Regulationen für Dienstleistungsqualität, so unverzichtbar sie sind, zeitigen nur begrenzte Effekte, wenn sie nicht im Arbeitsalltag eine Fundierung in der Kompetenz und im Berufsethos der Dienstleister finden.

Auf die Frage, wer der Träger einer auf mehr Autonomie in der Arbeit setzenden Professionalität sein könne, war die
Antwort der klassischen angelsächsischen Professions-Theorie: Berufsverbände bzw. -kammern. Diese Antwort aber bezog sich im Wesentlichen auf die freien akademischen Berufe (Ärzte, Rechtsanwälte u.a.). Für abhängig Beschäftigte ist sie nicht geeignet, auch wenn sich viele Merkmale ihrer Arbeit derjenigen der Professionen annähern. Als abhängig Beschäftigte brauchen sie Organisationsformen, die professionelle Standards in Betrieben über Instrumente von Tarif- und Rahmentarifverträgen durchsetzen und ihre Einhaltung verbindlich kontrollieren können. Berufsverbände können das nicht leisten.
Es bleiben in der deutschen Situation also vor allem die Dienstleistungsgewerkschaften. Aber auch sie können die Entwicklung und Sicherung professioneller Standards in der Arbeit nur leisten, wenn sie ihre Politik nicht allein auf Arbeitsbedingungen ausrichten, sondern die spezifischen Bedürfnisse der unterschiedlichen Berufsgruppen ernst nehmen und einem umfassenden Professionalisierungskonzept folgen, das auch die Verbindung von professionellem Zuschnitt der Dienstleistungsarbeit und Dienstleistungsqualität für Kunden/Klienten vor der Öffentlichkeit sichtbar machen kann.

\section{LITERATUR}

\section{Arbeitsgemeinschaft Qualifikations-Entwicklungs-Management (QUEM) (Hrsg.) (verschiedene Jahrgänge): Kompetenzentwicklung (1996-2005), Münster/New York/Berlin}

Autorengruppe Bildungsberichterstattung (2008): Bildung in Deutschland 2008, Bielefeld

Avey, J.B./Luthans, F./Smith, M./Palmer, N.F. (2010): Impact of Positive Psychological Capital on Employee Well-Being Over Time, in: Journal of Occupational Health Psychology 15 (1), S.17-28

Badura, B./Gross, P. (1976): Sozialpolitische Perspektiven, München Baethge, M. (2010): Neue soziale Segmentationsmuster in der beruflichen Bildung, in: Krüger, H.-H./Rabe-Kleberg, U./Kramer, R.-T./Budde, J. (Hrsg.): Bildungsungleichheit revisited, Wiesbaden, S. 275-298 Baethge, M. (2011): Beschäftigung und Arbeit in der nachindustriellen Gesellschaft, in: Otto, H-U./Thiersch, H. (Hrsg.): Handbuch Soziale Arbeit, 4. völlig neu bearbeitete Auflage, München, S. 143-161

Baethge, M./Achtenhagen, F./Arends, L./Babic, E./Baethge-Kinsky, V./Weber, S. (200): Berufsbildungs-PISA-Machbarkeitsstudie, Stuttgart Baethge, M./Arends, L. (2009): Feasibility Study VET-LSA. A comparative analysis of occupational profiles and VET programmes in 8 European countries - international report

Baethge, M./Oberbeck, H. (1986): Zukunft der Angestellten, Frankfurt a. M./New York

Baethge, M./Wilkens, I. (2001): "Goldenes Zeitalter" - "Tertiäre Krise": Perspektiven von Dienstleistungsbeschäftigung zum Beginn des 21. Jahrhunderts, in: Dies. (Hrsg.): Die große Hoffnung für das 21. Jahrhundert?, Opladen

Baethge-Kinky, V./Holm, R./Tullius, K. (2006): Dynamische Zeiten langsamer Wandel: Betriebliche Kompetenzentwicklung von Fachkräften in zentralen Tätigkeitsfeldern der deutschen Wirtschaft, Göttingen, Ms.

Bell, D. (1975): Die nachindustrielle Gesellschaft, Frankfurt, a. M.

(Amerikanische Ausgabe 1973: "The Coming of Post-Industrial Society ", New York)

Böhle, F./Voß, G./Wachtler, G. (2010): Handbuch Arbeitssoziologie, Wiesbaden

Breisig, Th./König, S./Rehling, M./Ebeling, M. (2010): "Sie müssen es nicht verstehen, Sie müssen es nur verkaufen! “ Vertriebssteuerung in Banken, Berlin
Bundesinstitut für Berufsbildung (BIBB)/Bundesanstalt für Arbeitsschutz und Arbeitsmedizin (BAuA) (2006.): Erwerbstätigenbefragung 2006 - Arbeit und Beruf im Wandel, Erwerb und Verwertung beruflicher Qualifikationen, www.bibb.de/de/26738htm

Dunkel, W./Weihrich, M. (2010): Arbeit als Interaktion, in: Böhle, F./ Voß, G./Wachtler, G. (Hrsg.): Handbuch Arbeitssoziologie, Wiesbaden, S. $177-200$

Gross, P. (1983): Die Verheißung der Dienstleistungsgesellschaft. Soziale Befreiung oder Sozialherrschaft?, Opladen

Hall, A. (2007): Tätigkeiten, berufliche Anforderungen und Qualifikationsniveau in Dienstleistungsberufen, in: Walden, G. (Hrsg.): Qualifizierungsentwicklung im Dienstleistungsbereich, Bonn, S. 153-208

Helmrich, R./Zika, G. (2010): BIBB/IAB-Qualifikations- und Berufshauptfeldprojektionen, Frankfurt a. M., Ms.

Kohn, M. (1977): Class and conformity: a study in values with reassessment, Chicago

Nonaka, I./Takeuchi, H. (1997): Die Organisation des Wissen, Frankfurt a. M./New York

Oberbeck, H. (2001): Zum Verhältnis von Dienstleistungsqualität und Dienstleistungsbeschäftigung, in: Baethge, M./Wilkens, I. (Hrsg.): Die große Hoffnung für das 21. Jahrhundert?, Opladen, S. 71-84

Polanyi, M. (1985): Implizites Wissen (dt. Ausg. von „The Tacit Dimension“), Frankfurt a. M.

Popitz, H./Bahrdt, H.P./Jüres, E.A./Kesting, H. (1957): Technik und Industriearbeit, Tübingen

Riese, H. (1997): Die Entwicklung des Bedarfs an Hochschulabsolventen in der Bundesrepublik Deutschland, Wiesbaden

Tiemann, M./Schade, H.J./Helmrich, R./Hall, A./Braun, U./Bott, P. (2008): Berufsfeld-Definitionen des BIBB auf Basis der Klassifikation der Berufe 1992, Bundesinstitut für Berufsbildung, Wissenschaftliche Diskussionspapiere (105), Bonn

Walden, G. (2007): Qualifikationsentwicklung im Dienstleistungsbereich, Bonn 